\title{
Regional Distribution of Different Types of Human Papillomavirus in Cervixes of Chinese Women: A Meta-analysis
}

\author{
Yue Wang ${ }^{1}$, Kunpeng Zhang ${ }^{1}$, Yali Hu ${ }^{1}$, Hongmei Ma ${ }^{1}$, Xiuyan Zheng ${ }^{1}$, Kelu Li ${ }^{1}$, Yanjuan Xie ${ }^{1}$, \\ Lijuan Pang ${ }^{1}$, Lin Tao ${ }^{1}$, Jianming $\mathrm{Hu}^{1}$, Yan $\mathrm{Qi}^{1}$, Feng $\mathrm{Li}^{2}$, Wenyi Gu${ }^{3}$, Hong Zou ${ }^{1, *}$ \\ ${ }^{1}$ Department of Pathology and Key Laboratory of Xinjiang Endemic and Ethnic Diseases (Ministry of Education), Shihezi University School of \\ Medicine, Shihezi, China \\ ${ }^{2}$ Department of Pathology, Beijing Chaoyang Hospital, Capital Medical University, Beijing, China \\ ${ }^{3}$ Australian Institute for Bioengineering and Nanotechnology (AIBN), University of Queensland, Brisbane, Australia
}

\section{Email address:}

zouhong_patho@163.com (Hong Zou)

${ }^{*}$ Corresponding author

\section{To cite this article:}

Yue Wang, Kunpeng Zhang, Yali Hu, Hongmei Ma, Xiuyan Zheng, Kelu Li, Yanjuan Xie, Lijuan Pang, Lin Tao, Jianming Hu, Yan Qi, Feng Li, Wenyi Gu, Hong Zou. Regional Distribution of Different Types of Human Papillomavirus in Cervixes of Chinese Women: A Meta-analysis. Journal of Cancer Treatment and Research. Vol. 6, No. 2, 2018, pp. 7-18. doi: 10.11648/j.jctr.20180602.11

Received: June 8, 2018; Accepted: June 26, 2018; Published: July 26, 2018

\begin{abstract}
Cervical cancer is a common gynecological malignancy that has shown a gradual increase in morbidity in recent years in China. High-risk human papillomaviruses (HR-HPVs) play a vital role in cervical cancer development and commercial vaccines are available. The overall HPV infection and geographical distribution characteristics of cervical Cancer among Chinese females have not been reported that may guide the application of the vaccines. This study systematically retrieves literature on HPV type and cervical cancer in China published from 2005 to 2017. Data are analyzed according to provinces and administrative areas for the meta-analysis. (1) SPSS17.0 software is used for the statistical data analysis. (2) $\chi^{2}$ inspection is also employed to analyze the infection rate of different HPV types of different cervical lesion groups in seven regions of China. Results show that China's HR-HPV16, 58, 18, 52, 33, 31, 53, 56, 59, 51, 35, and 68 infection rates are $60.49 \%, 11.16 \%, 9.68 \%$, $7.05 \%, 5.61 \%, 3.62 \%, 2.88 \%, 2.32 \%, 1.96 \%, 1.74 \%, 1.53 \%$, and $1.52 \%$, respectively. Differences in the HR-HPV infection rate of cervical cancer were observed among different areas, especially for HPV 31, 33, 52, and 58. The genotypes of the top five HR-HPV infection rates in cervical cancer also show differences in different regions. The results offer a basis for the prevention and treatment of cervical cancer in different parts of China. HPV type distribution in relation to cervical cancer varies among different regions and parts of China. Therefore, use of available or development of new vaccines suitable for regional types is necessary to improve the efficacy of the vaccines in preventing the primary HPV infection in different areas of China.
\end{abstract}

Keywords: China, Cervical Cancer, Human Papillomavirus, Meta-analysis

\section{Introduction}

Cervical cancer is one of the most common gynecologic malignant tumors and has become the most common cause of cancer-related deaths in women in developing countries, where approximately $85 \%$ of cases occur. Cervical cancer more frequently occurs in younger women and causes serious harm to women's health [1]. Unlike other tumors, cervical cancer has the characteristics of definite cause and longer reversing precancerous period. Thus, the early detection and timely treatment of cervical cancer may improve patient prognosis to some extent. Human papillomavirus (HPV) infection is an essential factor of cervical intraepithelial neoplasia (CIN) and cervical cancer. The HPV infection rate and distribution of HPV subtypes vary in different countries and regions. Infection of high-risk HPV (HR-HPV) infection (e.g., HPV 16, 18, 31, and 45) is the main cause of the occurrence and development of cervical cancer. Although the important HR-HPV types (e.g., HPV 16 and 18) show 
consistency in the geographic area distribution, the main HPV subtypes differ in different parts of the world [2, 3]. Hence, prevention and control measures with regional specificity to target infection of HPV subtypes are urgently needed.

China is the most populous country in the world with a high prevalence of cervical cancer. However, only a few studies with comprehensive analyses of the latest literature on the HPV infection condition of Chinese women have been conducted. To more comprehensively understand/map the geographic distribution of HPV types in different regions of China and relate the finding with HPV vaccine application. The current study analyzes literature on HR-HPV [4] infection of cervical cancer among Chinese women in different regions published in PubMed from 2005 to 2017. A meta-analysis was conducted to evaluate the status of HPV subtype infection in different Chinese regions. The results will provide evidence for vaccine application and other possible formulations of prevention and control measures that target the HPV subtype infection in various parts of China.

\section{Materials and Methods}

\subsection{Search Strategy and Eligibility Criteria}

As the primary data source, literature published from January 2005 to August 2017 were searched with the subject terms of "HPV", "cervical neoplasms/cervical cancer", "China"and their entry termsin the PubMed database. Two independent reviewers screened all articles by title and abstract based on theinclusion and exclusioncriteria. Duplicate records were automatically removed by a reference management software. Any disagreements between the two reviewers on paper selection were discussed by explicit selection rules, and fulltextswere reviewed if necessary. Fulltexts of eligible articles were retrieved and assessed by tworeviewers following the set processes.

\subsection{Inclusion and Exclusion Criteria for the Literature}

Inclusion criteria: (1) The research objects come from China, with clear source areas. The case number in a study includes the general screening patients, which may be suffering from cervical disease, precancerous lesions of the uterine cervix, and patients with cervical cancer. (2) The pathological or cytological classification of cervical lesions is definite, which includes invasive cervical cancer (ICC), high-level cervical intraepithelial neoplasia (HSIL), low-level cervical intraepithelial neoplasia (LSIL), and normal control group (i.e., healthy people and patients with cervicitis). Several studies may contain two or more pathological or cytological classifications. The ICC can be further divided into squamous cell carcinoma (SCC), glandular cell carcinoma or glands squamous cell carcinoma (ADC/ASC), and endometrial carcinoma (EC). HSIL is equivalent to the cervical intraepithelial neoplasia grade 2 (CIN2), cervical intraepithelial neoplasia grade 3 (CIN3), cervical glands intraepithelial neoplasia grade 2 (CGIN2), and cervical glands intraepithelial neoplasia grade 3 (CGIN3). Carcinoma in situ is classified as CIN3. LSIL is equivalent to the cervical intraepithelial neoplasia grade 1 (CIN1) and cervical glands intraepithelial neoplasia grade 1 (CGIN1). (3) The method for HPV classification is PCR. In this study, the specific case numbers of cervical lesions infection and infection of different HPV subtypes are connected. The essay methods should be sufficiently clear to introduce the HPV DNA detection process. The simple hybridization detection method based on non-amplification has been removed. (4) The minimum case number of each classification is 20. (5) If the article lacks specific information, then the authorsere contacted directly for specific details of the HPV classification data. If the data or partial data of the study were reported more than twice, then the largest sample size of the published article was included into the specific article.

Exclusion criteria: (1) Areview literature that lacksthe original source data. (2) Survey data that may have serious bias. (3) Experiment design in the original literature is not rigorous (e.g., the research data are incomplete). (4) Repeated case reports, vague data description, and investigation methods or data that do not conform to the research requirements.

\subsection{Data Excerpt}

The following key information serves as the excerpt for each paper: (1) The published year and journals name. (2) The specimen source (i.e., fresh or fixed tissue samples, cytology specimens, or their combination). (3) Pathological or cytological classification: the ICC (SCC/ADC/EC), HSIL (CIN 2/3, CGIN2/3), LSIL 1, CGIN1 (CIN), and normal control group (healthy crowd and cervicitis patients). (4) The total case number of each category and HR-HPV infection. 15 types of HR-HPV types were collected, namely, HPV 16, 18, $31,33,35,39,45,51,52,56,58,59,68,73,82$, and other probable high-risk types (e.g., HPV 53, 66, and83). (5) Area: Given the geographical distribution, the areas involved in this study are divided into East China (i.e., Shanghai, Jiangsu, Zhejiang, Anhui, Jiangxi, Shandong, Fujian, and Taiwan province), North China (i.e., Beijing, Tianjin, Shanxi, Hebei, and Central Inner Mongolia Autonomous Region), Central China (i.e., Henan, Hubei, and Hunan province), South China (i.e., Guangdong, Guangxi Zhuang Autonomous Region, Hainan province, the Hong Kong Special Administrative Region, and Macao Special Administrative Region), Southwest China (i.e., Chongqing, Sichuan, Guizhou, Yunnan Province, and the Tibet Autonomous Region), Northwest China (i.e., Shanxi, Gansu, Qinghai, Ningxia Hui Autonomous Region, Xinjiang Uygur Autonomous Region, Inner Mongolia Autonomous Region, and West La Shan Au), and Northeast China (i.e., Heilongjiang, Jilin, Liaoning, and East of Inner Mongolia).

\subsection{Statistical Analysis}

The general positive rate of HPV is the ratio of the positive numbers of HPV tests and included sample numbers. The different methods and strategies can detect different HPV 
types. Therefore, the infection rate of different HPV types were analyzed in the HPV positive samples of cervical cancer. The specific positive ratio of HPV types equals the ratio of HPV positive cases of specific types and sample cases that have the results of this specific type. Therefore, the sample size is different forestimating the diverse typesin terms of HPV-positive rates.

SPSS17.0 software is used for the statistical analysis of the data when weighing and combining the infection rates for the different studies. $\chi^{2}$ inspection is also employed to analyze the infection rate of different HPV types of different cervical lesion groups in seven regions of China to compare the HPV infection condition of different types.

\section{Results}

\subsection{Included Studies}

A total of 316 articles were retrieved from the PubMed database through the keyword search. Approximately 136 articles were initially selected by reviewing their titles and abstracts. Finally, 30 articles were selected after reviewing their full text based on the inclusion criteria (Figure 1, Table1) [5-34].
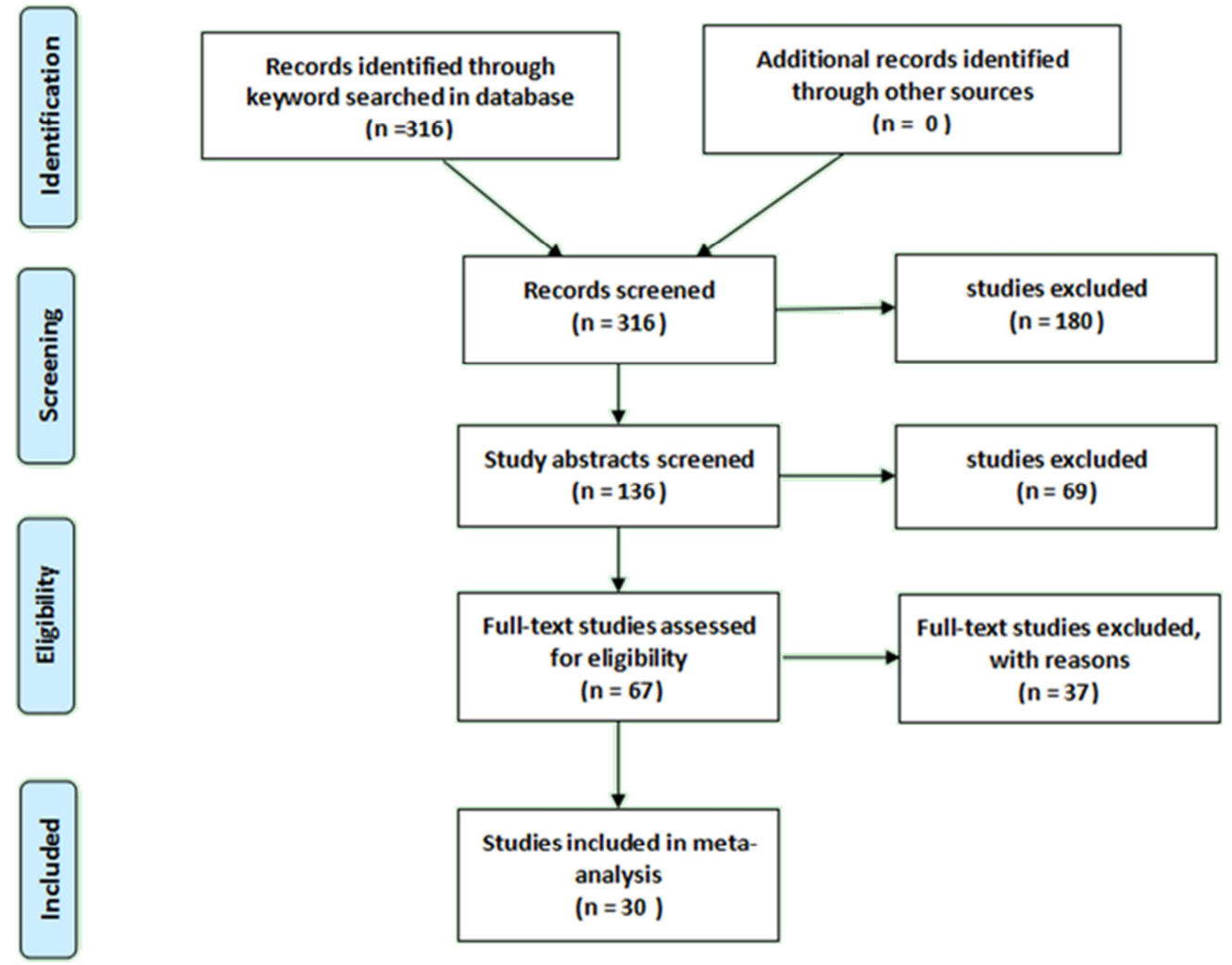

Figure 1. Flowchart showing the selection of articles included in the meta-analysis.

Table 1. The 30 selected studies and the detected HPV genotypes.

\begin{tabular}{|c|c|c|c|c|c|c|c|c|c|}
\hline Author & Year & Area & $\begin{array}{l}\text { No tissue } \\
\text { lesions } \\
\end{array}$ & LSIL & HSII & ICC & $\begin{array}{l}\text { Total number } \\
\text { of cases }\end{array}$ & $\begin{array}{l}\text { Detection } \\
\text { method }\end{array}$ & HPV genotypes \\
\hline Li J et $\mathrm{al}^{5}$. & 2011 & Chengdu & - & - & 63 & 144 & 207 & PCR & $16,18,31,33,35,45,51,52,58$ \\
\hline $\mathrm{Li} \mathrm{H}$ et $\mathrm{a}^{6}$. & 2013 & Hunan & 3115 & 165 & 147 & 78 & 3505 & PCR & $16,18,31,33,35,39,45,51,52,56,58,59,68$ \\
\hline Zhao Y et al ${ }^{7}$. & 2008 & Yianbian & 20 & 56 & 146 & 100 & 322 & PCR & $16,18,33,52,58$ \\
\hline Hong $\mathrm{D}$ et $\mathrm{al}^{8}$. & 2008 & Zhejiang & 217 & 105 & 345 & 181 & 848 & PCR & $16,18,31,33,39,45,51,52,56,58,59,68,82$ \\
\hline Wu EQ et $\mathrm{al}^{9}$. & 2009 & Mogolian & - & 71 & 27 & 77 & 175 & PCR & $16,18,31,33,45,52,58,59$ \\
\hline Cai HB et $\mathrm{al}^{10}$. & 2009 & Hubei & - & - & 60 & 112 & 172 & PCR & $16,18,31,33,35,52,58,59$ \\
\hline Li C et $\mathrm{al}^{11}$. & 2010 & Beijing & 233 & 295 & - & - & 528 & PCR & $16,18,31,33,35,39,45,51,52,56,58,59,68,73$ \\
\hline Sun ZR et $\mathrm{al}^{12}$. & 2010 & Liaoning & 165 & - & 117 & 154 & 436 & PCR & $16,18,31,33,35,39,45,51,52,56,58,59,68$ \\
\hline Liu $X$ et $\mathrm{al}^{13}$. & 2010 & Liaoning & - & 180 & 517 & 134 & 831 & PCR & $16,18,31,33,35,39,45,51,52,56,58,59,68$ \\
\hline Wu D et $\mathrm{al}^{14}$. & 2010 & Fujian & 314 & 129 & 102 & 96 & 641 & PCR & $16,18,31,33,35,39,45,51,52,56,58,59,68$ \\
\hline Yuan $\mathrm{X}$ et $\mathrm{al}^{15}$. & 2011 & Shandong & 471 & 302 & 518 & 198 & 1489 & PCR & $16,18,31,33,35,39,45,51,52,56,58,59,68$ \\
\hline LI J et $\mathrm{al}^{16}$. & 2012 & Western & - & 143 & 457 & - & 600 & PCR & $16,18,31,33,35,39,45,51,52,56,58,59,66,68$, \\
\hline Liu $\mathrm{W}$ et $\mathrm{al}^{17}$. & 2013 & Changchun & 40 & - & - & 65 & 105 & PCR & $16,18,31,33,35,39,45,51,52,56,58,59,68$ \\
\hline Yang L et al ${ }^{19}$. & 2013 & Daqing & 1702 & 24 & - & - & 1726 & PCR & $16,18,39,52,58$ \\
\hline Liu $X X$ et $\mathrm{al}^{20}$. & 2014 & Zhejiang & 13891 & 260 & 108 & - & 14259 & PCR & $16,18,31,33,39,45,51,52,56,58,59,68,73,82$ \\
\hline
\end{tabular}




\begin{tabular}{lllllllll}
\hline Author & Year & Area & $\begin{array}{l}\text { No tissue } \\
\text { lesions }\end{array}$ & LSIL & HSIl & ICC & $\begin{array}{l}\text { Total number Detection } \\
\text { of cases }\end{array}$ & method \\
mPV genotypes
\end{tabular}

ICC: invasive cervical cancer; HSIL: high grade intraepithelial neoplasia of cervix; LSIL: low-grade intraepithelial neoplasia of the cervix; control group:no lesions in the cervical tissue.

\subsection{Meta-analysis of the Total Infection Rate of HPV Genotypes in Cervixes}

The meta-analysis includes 84,724 cervical biopsies or specimens of exfoliated cells, namely, 4,525 ICC cases, 10,194 HSIL cases, 6,569 LSIL cases, and 63,436 control subject cases without cervical disease. The most common type is HPV 16. The infection rate of HPV 16 is $60.49 \%$ in ICC, $41.77 \%$ in HSIL, and $18.75 \%$ in LSIL. The infection rate in the normal cervical tissues is $2.39 \%$.

The HPV genotypes in ICC with infection rates of more than $2 \%$ are HPV $16,58,18,52,33,31,53$, and 56 . The infection rates of these genotypes are $60.49 \%, 11.16 \%, 9.68 \%$, $7.05 \%, 5.61 \%, 3.62 \%, 2.88 \%$, and $2.32 \%$ respectively. The
HPV genotypes in HSIL with infection rates of more than 2\% are HPV 16, 58, 52, 33, 31, 53, 18, 56, 66, 51, 39, 59, 68, and 35 , with infection rates of $41.77 \%, 20.29 \%, 15.31 \%, 12.04 \%$, $7.98 \%, 6.65 \%, 5.94 \%, 5.59 \%, 5.58 \%, 5.35 \%, 3.22 \%, 2.12 \%$, $2.11 \%$, and $2.02 \%$, respectively. The HPV genotypes in LSIL with an infection rate of more than $2 \%$ are HPV 52, 16, 53, 58, $33,56,18,51,31,39,66,68,35$, and 59 . The infection rates of these genotypes are $19.95 \%, 18.75 \%, 16.99 \%, 12.73 \%, 8.31 \%$, $7.24 \%, 6.92 \%, 6.30 \%, 5.85 \%, 5.38 \%, 5.37 \%, 4.66 \%, 3.10 \%$, and $2.63 \%$, respectively. The HPV genotypes in normal cervixes with infection rates of more than $1 \%$ are HPV 52, 16 , 58,53 , and 83 , with infection rates of $2.55 \%, 2.39 \%, 2.35 \%$, $1.10 \%$, and $1.02 \%$, respectively (Table 2 and Figure 2).

Table 2. Infection rates of different genotypes in different cervical lesions of the Chinese population.

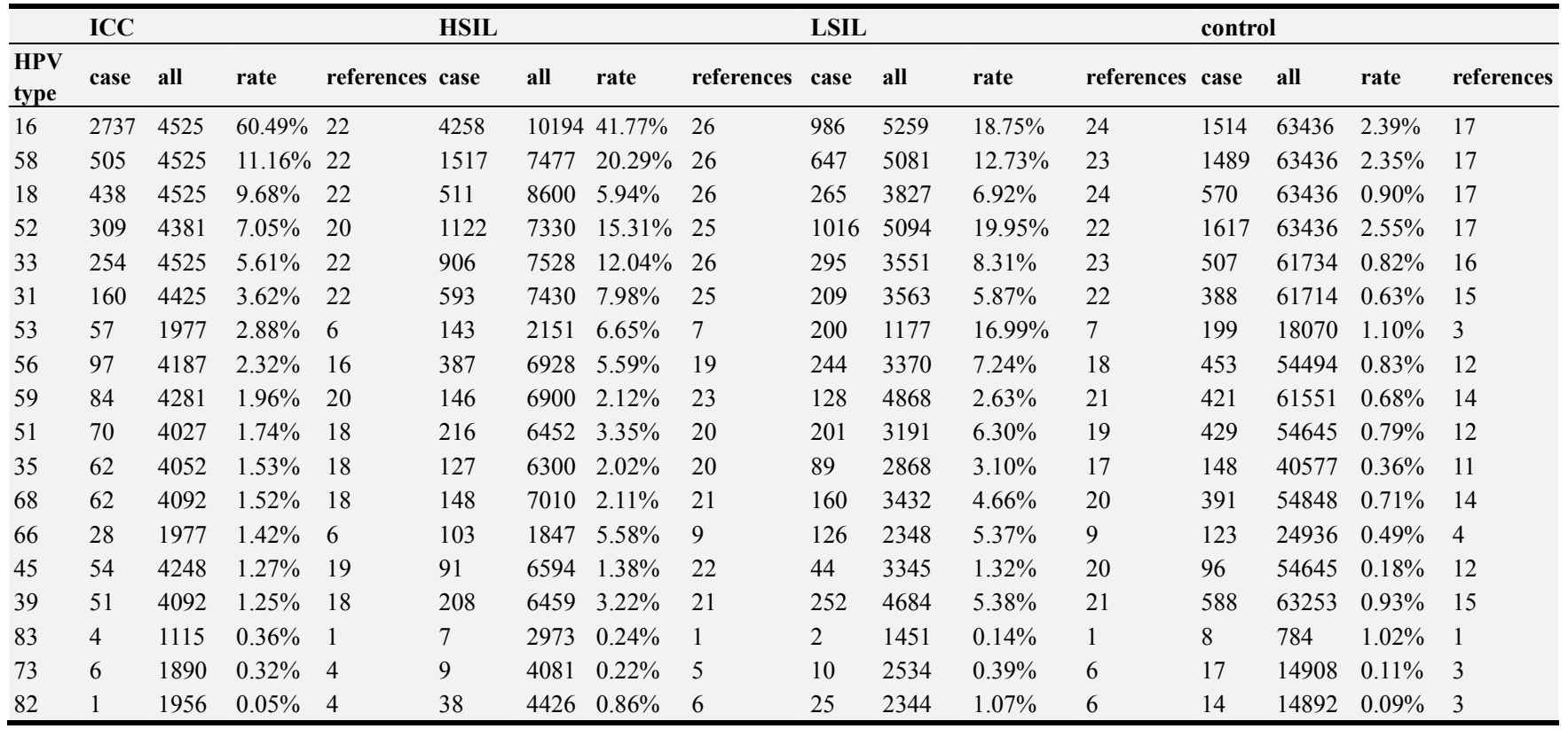

This table shows the Chinese women infected by different HPV genotypesin ICC, HSIL, LSIL, and cervical tissue without lesions. The cases were sorted according to the ranking of infection rates of different HPVgenotypes in ICC.

ICC: invasive cervical cancer; HSIL: high grade intraepithelial neoplasia of cervix; LSIL: low-grade intraepithelial neoplasia of the cervix; control group:no lesions in the cervical tissue. 

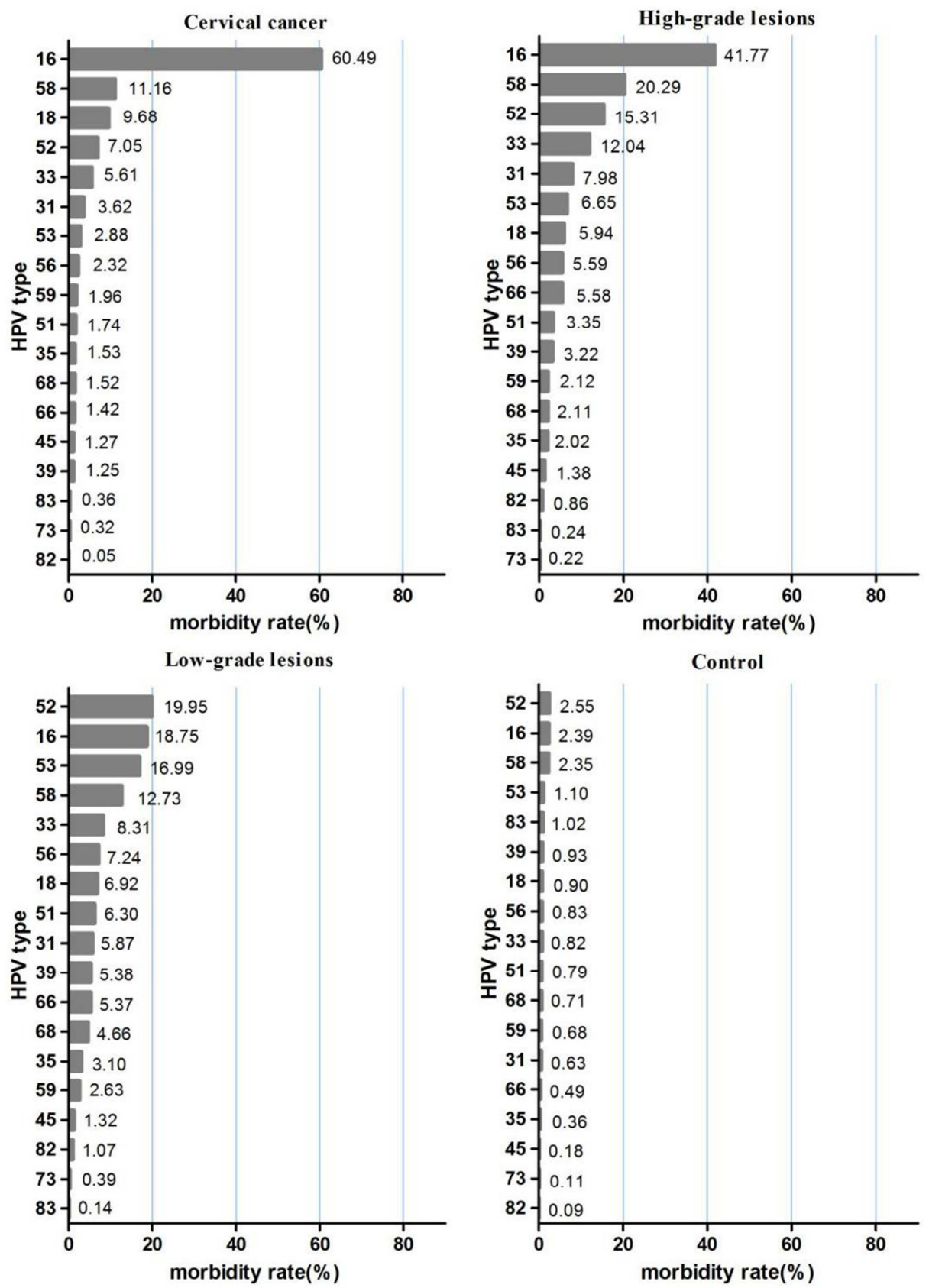

Figure 2. Prevalence of different genotypes in different cervical lesions in the whole China. ICC: invasive cervical cancer; HSIL: high grade intraepithelial neoplasia of cervix; LSIL: low-grade intraepithelial neoplasia of the cervix; control group:no lesions in the cervical tissue. The figure shows the prevalence of different HPV genotypes in ICC, HSIL, LSIL, and the control group in China, where the HPV16 infection rate is the highest.

\subsection{Meta-analysis of the Top Five High-Risk Types of Cervical Cancer in Seven Regions of China}

Articles for Northeast China, North China, East China, South China, Central China, Northwest China, Southwest Chinawere obtained. A total of eight HR-HPV genotypes (i.e., HPV 16, 18, 31, 33, 52, 58, 59, and 68) of cervical cancer were selected by synthetically analyzing the top five high-risk types of cervical cancer in different regions. Table 3 shows that the top five genotypes of ICC in the seven regions are inconsistent. The top five HPV genotypes in the seven regions of China were generally HPV 16, 18, 31, 33, 52, 58, 59 and 68 . A histogram was made to discuss the distribution of HPV subtypes in the control group (intraepithelial neoplasia) and cervical lesions (cervical cancer) (Table 3 and Figure 3). Figure 2 shows that HPV 16 is the most widely distributed in the seven regions. Its infection rate is $75.56 \%$ in South China, $70.12 \%$ in North China, $70.00 \%$ in Central China, $63.13 \%$ in Northeast China, 62.77\% in Southwest China, 55.07\% in Northwest China, and 51.42\% in East China. HPV 16, 18, 52 and 58 were included in the top five HPV genotype infection rates of cervical cancer in the seven regions, which indicates that they were the dominant types. These types were followed by HPV 31, 33, 59, and 68 . Figure 3 also shows the top five high-risk infection rates. 

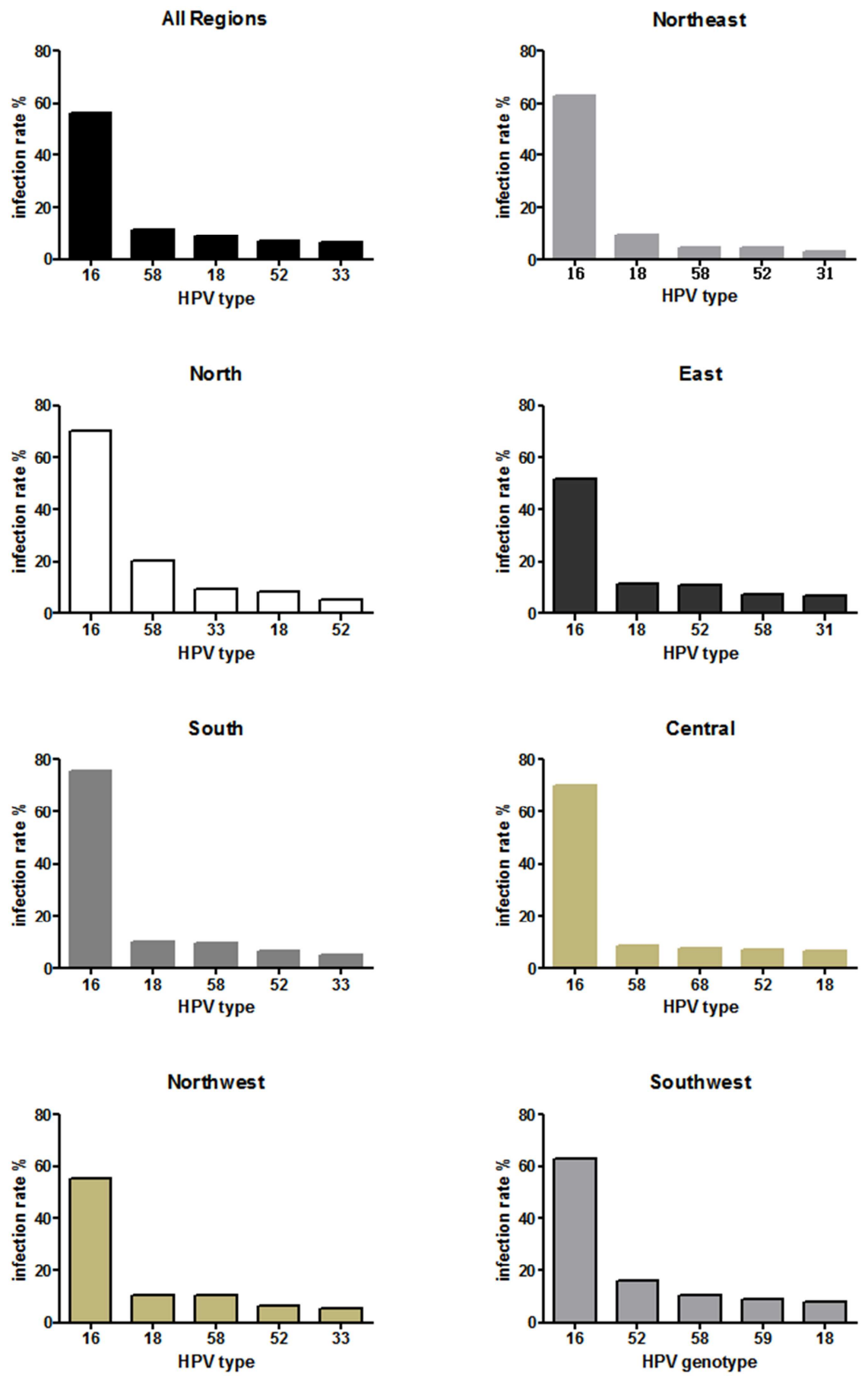

Figure 3. Infection rate of the top five genotypes among the seven regions of China.

Table 3. HPV genotypes of the top five genotypes in the seven regions.

\begin{tabular}{|c|c|c|c|c|c|c|c|c|c|c|c|c|c|}
\hline \multirow{2}{*}{ Region } & \multirow{2}{*}{$\begin{array}{l}\text { HPV } \\
\text { type }\end{array}$} & \multicolumn{3}{|l|}{$\mathrm{CC}$} & \multicolumn{3}{|l|}{ HSIL } & \multicolumn{3}{|l|}{ LSIL } & \multicolumn{3}{|c|}{ control } \\
\hline & & case & all & rate & case & all & rate & case & all & rate & case & all & rate \\
\hline \multirow[t]{5}{*}{ Northeast } & 16 & 286 & 453 & $63.13 \%$ & 431 & 780 & $55.26 \%$ & 70 & 260 & $26.92 \%$ & 39 & 1927 & $2.02 \%$ \\
\hline & 18 & 44 & 453 & $9.71 \%$ & 29 & 780 & $3.72 \%$ & 11 & 260 & $4.23 \%$ & 13 & 1927 & $0.67 \%$ \\
\hline & 58 & 21 & 453 & $4.64 \%$ & 94 & 780 & $12.05 \%$ & 37 & 260 & $14.23 \%$ & 15 & 1927 & $0.78 \%$ \\
\hline & 52 & 19 & 453 & $4.19 \%$ & 51 & 780 & $6.54 \%$ & 39 & 260 & $15.00 \%$ & 24 & 1927 & $1.25 \%$ \\
\hline & 31 & 11 & 353 & $3.12 \%$ & 53 & 634 & $8.36 \%$ & 12 & 180 & $6.67 \%$ & 1 & 205 & $0.49 \%$ \\
\hline \multirow[t]{3}{*}{ North } & 16 & 730 & 1041 & $70.12 \%$ & 1012 & 2090 & $48.42 \%$ & 110 & 839 & $13.11 \%$ & 382 & 18402 & $2.08 \%$ \\
\hline & 33 & 95 & 1041 & $9.13 \%$ & 186 & 2090 & $8.90 \%$ & 27 & 839 & $3.22 \%$ & 82 & 18402 & $0.45 \%$ \\
\hline & 18 & 84 & 1041 & $8.07 \%$ & 127 & 2090 & $6.08 \%$ & 18 & 839 & $2.15 \%$ & 162 & 18402 & $0.88 \%$ \\
\hline
\end{tabular}




\begin{tabular}{|c|c|c|c|c|c|c|c|c|c|c|c|c|c|}
\hline \multirow{2}{*}{ Region } & \multirow{2}{*}{$\begin{array}{l}\text { HPV } \\
\text { type }\end{array}$} & \multicolumn{3}{|l|}{$\mathrm{CC}$} & \multicolumn{3}{|l|}{ HSIL } & \multicolumn{3}{|c|}{ LSIL } & \multicolumn{3}{|c|}{ control } \\
\hline & & case & all & rate & case & all & rate & case & all & rate & case & all & rate \\
\hline & 52 & 52 & 1041 & $5.00 \%$ & 207 & 2090 & $9.90 \%$ & 52 & 839 & $6.20 \%$ & 564 & 18402 & $3.06 \%$ \\
\hline \multirow[t]{5}{*}{ East } & 16 & 524 & 1019 & $51.42 \%$ & 768 & 1817 & $42.27 \%$ & 222 & 1172 & $18.94 \%$ & 386 & 14893 & $2.59 \%$ \\
\hline & 18 & 113 & 1019 & $11.09 \%$ & 53 & 1817 & $2.92 \%$ & 43 & 1172 & $3.67 \%$ & 171 & 14893 & $1.15 \%$ \\
\hline & 52 & 112 & 1019 & $10.99 \%$ & 233 & 1817 & $12.82 \%$ & 176 & 1172 & $15.02 \%$ & 296 & 14893 & $1.99 \%$ \\
\hline & 58 & 71 & 1019 & $6.97 \%$ & 203 & 1817 & $11.17 \%$ & 117 & 1172 & $9.98 \%$ & 294 & 14893 & $1.97 \%$ \\
\hline & 31 & 69 & 1019 & $6.77 \%$ & 178 & 1817 & $9.80 \%$ & 89 & 1172 & $7.59 \%$ & 160 & 14893 & $1.07 \%$ \\
\hline \multirow[t]{5}{*}{ South } & 16 & 170 & 225 & $75.56 \%$ & 211 & 594 & $35.52 \%$ & 43 & 245 & $17.55 \%$ & 35 & 6866 & $0.51 \%$ \\
\hline & 18 & 23 & 225 & $10.22 \%$ & 36 & 594 & $6.06 \%$ & 12 & 99 & $12.12 \%$ & 16 & 6866 & $0.23 \%$ \\
\hline & 58 & 22 & 225 & $9.78 \%$ & 98 & 594 & $16.50 \%$ & 127 & 1555 & $8.17 \%$ & 31 & 6866 & $0.45 \%$ \\
\hline & 52 & 15 & 225 & $6.67 \%$ & 25 & 369 & $6.78 \%$ & 228 & 1548 & $14.73 \%$ & 78 & 6866 & $1.14 \%$ \\
\hline & 33 & 11 & 225 & $4.89 \%$ & 57 & 594 & $9.60 \%$ & 23 & 87 & $26.44 \%$ & 31 & 6866 & $0.45 \%$ \\
\hline \multirow[t]{5}{*}{ Central } & 16 & 133 & 190 & $70.00 \%$ & 100 & 207 & $48.31 \%$ & 21 & 165 & $12.73 \%$ & 89 & 3115 & $2.86 \%$ \\
\hline & 58 & 16 & 190 & $8.42 \%$ & 23 & 207 & $11.11 \%$ & 16 & 165 & $9.70 \%$ & 67 & 3115 & $2.15 \%$ \\
\hline & 68 & 6 & 78 & $7.69 \%$ & 1 & 147 & $0.68 \%$ & 2 & 165 & $1.21 \%$ & 25 & 3115 & $0.80 \%$ \\
\hline & 52 & 13 & 190 & $6.84 \%$ & 10 & 207 & $4.83 \%$ & 27 & 165 & $16.36 \%$ & 97 & 3115 & $3.11 \%$ \\
\hline & 18 & 12 & 190 & $6.32 \%$ & 12 & 207 & $5.80 \%$ & 4 & 165 & $2.42 \%$ & 36 & 3115 & $1.16 \%$ \\
\hline \multirow[t]{5}{*}{ Northwest } & 16 & 776 & 1409 & $55.07 \%$ & 276 & 945 & $29.21 \%$ & 196 & 673 & $29.12 \%$ & 192 & 1102 & $17.42 \%$ \\
\hline & 18 & 147 & 1409 & $10.43 \%$ & 74 & 945 & $7.83 \%$ & 48 & 673 & $7.13 \%$ & 55 & 1102 & $4.99 \%$ \\
\hline & 58 & 144 & 1409 & $10.22 \%$ & 132 & 945 & $13.97 \%$ & 101 & 673 & $15.01 \%$ & 113 & 1102 & $10.25 \%$ \\
\hline & 52 & 91 & 1409 & $6.46 \%$ & 124 & 945 & $13.12 \%$ & 110 & 673 & $16.34 \%$ & 145 & 1102 & $13.16 \%$ \\
\hline & 33 & 74 & 1409 & $5.25 \%$ & 94 & 945 & $9.95 \%$ & 56 & 673 & $8.32 \%$ & 75 & 1102 & $6.81 \%$ \\
\hline \multirow[t]{5}{*}{ Southeast } & 16 & 118 & 188 & $62.77 \%$ & 1460 & 3761 & $38.82 \%$ & 324 & 1905 & $17.01 \%$ & 391 & 17131 & $2.28 \%$ \\
\hline & 52 & 7 & 44 & $15.91 \%$ & 472 & 1122 & $42.07 \%$ & 384 & 437 & $87.87 \%$ & 413 & 17131 & $2.41 \%$ \\
\hline & 58 & 20 & 188 & $10.64 \%$ & 491 & 1044 & $47.03 \%$ & 191 & 417 & $45.80 \%$ & 544 & 17131 & $3.18 \%$ \\
\hline & 59 & 4 & 44 & $9.09 \%$ & 39 & 838 & $4.65 \%$ & 49 & 302 & $16.23 \%$ & 40 & 16968 & $0.24 \%$ \\
\hline & 18 & 15 & 188 & $7.98 \%$ & 180 & 2167 & $8.31 \%$ & 129 & 619 & $20.84 \%$ & 117 & 17131 & $0.68 \%$ \\
\hline
\end{tabular}

This table shows the prevalence of the top five HPV genotypes among seven regions of China, namely, include Northeast China, North China, East China, South China, Central China, Northwest China and Southwest China.

\subsection{Comparison of the Infection Rate Difference of the Eight Genotypes in the Seven Regions}

The chi-square test of $\mathrm{R} \times \mathrm{C}$ table in the SPSS statistical methods were utilized to compare the multiple sample rates. Finally, the chi-square segmentation method was implemented to readjust the inspection level of the alpha value [35]. The adjusted inspection level alpha was $\alpha^{\natural}=0.002273$. The results are listed in Table 4.

The highest infection rate of HPV 16 is $75.56 \%$ in South China, which shows significant differences with the infection rates in Northeast China $\left(63.13 \%, p=0.001<\alpha^{\prime}\right)$, East China $\left(51.42 \%, \mathrm{p}=0.000<\alpha^{\prime}\right)$ and Northwest China $(55.07 \%, \mathrm{p}=$ $0.001<\alpha$ '); the infection rates in North China and Central China are $70.12 \%$ and $70.00 \%$, which show significant differences compared with the infection rates of East China $(51.42 \%, \mathrm{p}=$ $\left.0.000<\alpha^{\prime}\right)$ and Northwest China $\left(55.07 \%, \mathrm{p}=0.000<\alpha^{\prime}\right)$. Moreover, the infection rate in Northeast China is $63.13 \%$, which shows significant difference with the infection rate in East China $\left(51.42 \%, \mathrm{p}=0.001<\alpha^{\prime}\right)$. The highest infection rate of HPV 31 is $6.77 \%$ in East China, which shows significant difference with the infection rates in North China $(3.36 \%, \mathrm{p}=$ $\left.0.000<\alpha^{\prime}\right)$, Northwest China $\left(2.56 \%, p=0.000<\alpha^{\prime}\right)$, and Southwest China $\left(0 \%, p=0.000<\alpha^{\prime}\right)$. The highest infection rate of HPV33 is $9.13 \%$ in North China, which is significantly different from the infection rates of Northeast China $(2.87 \%, \mathrm{p}$ $\left.=0.000<\alpha^{\prime}\right)$, East China $\left(5.59 \%, p=0.002<\alpha^{\prime}\right)$, Central China $\left(1.58 \%, \mathrm{p}=0.000<\alpha^{\prime}\right)$, Northwest China $(5.25 \%, \mathrm{p}=0.002$ $\left.<\alpha^{\prime}\right)$, and Southwest China $\left(0.53 \%, \mathrm{p}=0.000<\alpha^{\prime}\right)$. The highest infection rate of HPV 52 is $15.91 \%$ in Southwest China, which shows significant differences with the infection rates in
Northeast China $\left(4.19 \%, \mathrm{p}=0.001<\alpha^{\prime}\right)$ and North China $(5.00 \%, p=0.002<\alpha)$; the infection rate in East China is $10.99 \%$, which shows significant differences with the infection rates of Northeast China $(4.19 \%, p=0.000<\alpha$ ), North China $\left(5.00 \%, \mathrm{p}=0.000<\alpha^{\prime}\right)$, and Northwest China $(6.49 \%, \mathrm{p}=0.001$ $\left.<\alpha^{\prime}\right)$. The highest infection rate of HPV 58 is $20.27 \%$ in North China, which shows significant differences with the infection rates in Northeast China $\left(4.64 \%, \mathrm{p}=0.000<\alpha^{\prime}\right)$, East China $\left(6.97 \%, \mathrm{p}=0.000<\alpha^{\prime}\right)$, South China $\left(9.78 \%, \mathrm{p}=0.000<\alpha^{\prime}\right)$, Central China $\left(8.42 \%, p=0.000<\alpha^{\prime}\right)$, Northwest China $\left(10.22 \%, p=0.000<\alpha^{\prime}\right)$, and Southwest China $(10.64 \%, p=$ $\left.0.002<\alpha^{\prime}\right)$; the infection rate in Northwest China is $10.22 \%$, which also shows significant differences with the infection rate of Northeast China $\left(4.64 \%, p=0.000<\alpha^{\prime}\right)$. In addition, the highest infection rate of HPV 59 is $9.09 \%$ in Southwest China, which shows significant differences with the infection rates in Northeast China $\left(0.85 \%, p=0.000<\alpha^{\prime}\right)$, Central China $(1.05 \%$, $\left.\mathrm{p}=0.002<\alpha^{\prime}\right)$, and Northwest China $\left(0.92 \%, \mathrm{p}=0.000<\alpha^{\prime}\right)$; the infection rate in North China is $3.27 \%$, which shows significant differences with the infection rate in Northwest China $(0.92 \%, p=0.000<\alpha$ '). The highest infection rate of HPV 68 is $7.69 \%$ in Central China, which shows significant differences with the infection rates in Northeast China $(1.42 \%$, $\left.\mathrm{p}=0.001<\alpha^{\prime}\right)$, North China $\left(1.35 \%, \mathrm{p}=0.000<\alpha^{\prime}\right)$, East China $\left(0.39 \%, \mathrm{p}=0.000<\alpha^{\prime}\right)$, and South China $(0.89 \%, \mathrm{p}=0.001$ $\left.<\alpha^{\prime}\right)$; the infection rate in Northwest China is $2.27 \%$, which shows significant differences with the infection rate in East China $\left(0.39 \%, p=0.001<\alpha^{\prime}\right)$.

The infection rates of HPV 18 genotype showed no statistical differences among the different regions of China. 
Table 4. Comparison of the infection rates of the different genotypes among the seven regions.

\begin{tabular}{|c|c|c|c|c|c|c|c|c|c|c|c|c|c|c|c|}
\hline Region & & $\begin{array}{l}\text { HPV } \\
16 \%\end{array}$ & $\mathbf{P}$ & $\begin{array}{l}\text { HPV } \\
31 \%\end{array}$ & $\mathbf{P}$ & $\begin{array}{l}\text { HPV } \\
33 \%\end{array}$ & $\mathbf{P}$ & $\begin{array}{l}\text { HPV } \\
52 \%\end{array}$ & $\mathbf{P}$ & $\begin{array}{l}\text { HPV } \\
58 \%\end{array}$ & $\mathbf{P}$ & $\begin{array}{l}\text { HPV } \\
59 \%\end{array}$ & $\mathbf{P}$ & $\begin{array}{l}\text { HPV } \\
68 \%\end{array}$ & $\mathbf{P}$ \\
\hline \multirow[t]{6}{*}{ Northeast } & & 63.13 & & 3.12 & & 2.87 & & 4.19 & & 4.64 & & 0.85 & & 1.42 & \\
\hline & North & 70.12 & & 3.36 & & 9.13 & $* * *$ & 5.00 & & 20.27 & $* * *$ & 3.27 & & 1.35 & \\
\hline & East & 51.42 & $* * *$ & 6.77 & & 5.59 & & 10.99 & $* * *$ & 6.97 & & 2.36 & & 0.39 & \\
\hline & South & 75.56 & $* *$ & 1.78 & & 4.89 & & 6.67 & & 9.78 & & 1.78 & & 0.89 & \\
\hline & Central & 70.00 & & 2.63 & & 1.58 & & 6.84 & & 8.42 & & 1.05 & & 7.69 & $* *$ \\
\hline & Northwest & 55.07 & & 2.56 & & 5.25 & & 6.46 & & 10.22 & $* * *$ & 0.92 & & 2.27 & \\
\hline \multirow[t]{6}{*}{ North } & & 70.12 & & 3.36 & & 9.13 & & 5.00 & & 20.27 & & 3.27 & & 1.35 & \\
\hline & East & 51.42 & $* * *$ & 6.77 & $* * *$ & 5.59 & * & 10.99 & $* * *$ & 6.97 & $* * *$ & 2.36 & & 0.39 & \\
\hline & South & 75.56 & & 1.78 & & 4.89 & & 6.67 & & 9.78 & $* * *$ & 1.78 & & 0.89 & \\
\hline & Central & 70.00 & & 2.63 & & 1.58 & $* * *$ & 6.84 & & 8.42 & $* * *$ & 1.05 & & 7.69 & $* * *$ \\
\hline & Northwest & 55.07 & $* * *$ & 2.56 & & 5.25 & $* * *$ & 6.46 & & 10.22 & $* * *$ & 0.92 & $* * *$ & 2.27 & \\
\hline & Southwest & 62.77 & & 0.00 & & 0.53 & $* * *$ & 15.91 & $*$ & 10.64 & $*$ & 9.09 & & 0.00 & \\
\hline \multirow[t]{3}{*}{ East } & & 51.42 & & 6.77 & & 5.59 & & 10.99 & & 6.97 & & 2.36 & & 0.39 & \\
\hline & Northwest & 55.07 & & 2.56 & $* * *$ & 5.25 & & 6.46 & $* * *$ & 10.22 & & 0.92 & & 2.27 & $* * *$ \\
\hline & Southwest & 62.77 & & 0.00 & $* * *$ & 0.53 & & 15.91 & & 10.64 & & 9.09 & & 0.00 & \\
\hline \multirow[t]{4}{*}{ South } & & 75.56 & & 1.78 & & 4.89 & & 6.67 & & 9.78 & & 1.78 & & 0.89 & \\
\hline & Central & 70.00 & & 2.63 & & 1.58 & & 6.84 & & 8.42 & & 1.05 & & 7.69 & $* *$ \\
\hline & Northwest & 55.07 & $* * *$ & 2.56 & & 5.25 & & 6.46 & & 10.22 & & 0.92 & & 2.27 & \\
\hline & Southwest & 62.77 & & 0.00 & & 0.53 & & 15.91 & & 10.64 & & 9.09 & & 0.00 & \\
\hline \multirow[t]{3}{*}{ Central } & & 70.00 & & 2.63 & & 1.58 & & 6.84 & & 8.42 & & 1.05 & & 7.69 & \\
\hline & Northwest & 55.07 & $* * *$ & 2.56 & & 5.25 & & 6.46 & & 10.22 & & 0.92 & & 2.27 & \\
\hline & Southwest & 62.77 & & 0.00 & & 0.53 & & 15.91 & & 10.64 & & 9.09 & $*$ & 0.00 & \\
\hline \multirow[t]{2}{*}{ Northwest } & & 55.07 & & 2.56 & & 5.25 & & 6.46 & & 10.22 & & 0.92 & & 2.27 & \\
\hline & Southwest & 62.77 & & 0.00 & & 0.53 & & 15.91 & & 10.64 & & 9.09 & $* * *$ & 0.00 & \\
\hline
\end{tabular}

This table shows the comparison of infection rates of the different genotypes among the seven regions.

$*: \mathrm{p}=0.002 * *: \mathrm{p}=0.001 * * *: \mathrm{p}=0.000$.

\section{Discussion}

The International Agency for Research on Cancer (IARC) of the World Health Organization has performed meta-analyses of HPV distribution in cervical cancer around the world since 2003 and regularly updates its data [36-38]. Results show that respective preponderant HPV types exist in different populations and regions, which indicate that regional differences existed in HPV prevention and treatment. The latest report concerning HPV distribution in mainland China was published in 2009 [39]. However, the data have not been updated, and the regional differences in the HPV types have not been analyzed in detail. China is the world's most populous country with a population of 1.36 billion, which accounts for approximately $18.84 \%$ of the world population. Therefore, determiningthe HPV infection status of the Chinese population is significant and important. Analysis of the HPV infection status of cervical cancer and associated lesions in Chinese population has also become necessary because of the different geographical types, races, and lifestyles in different regions of China. The latest summary of meta-analyses in existing literature [39] was published in 2009, and the number of people and cases included in this summary is relatively rare. The current study analyzes and summarizes the HPV infection studies in cervical lesions published from 2005 to 2017. A total of 84,414 cervical biopsies or exfoliated cells were included in this study, namely, 4,525 ICC cases,
10,194 HSIL cases, 5,259 LSIL cases, and 63,436 control subject cases without cervical lesions. This meta-analysis has the highest number of samples included to date, which may provide evidence for the prevention and treatment of cervical cancer and precancerous lesions for the Chinese people. In this study, the eight most prevalent of HPV genotypes in ICC and HSIL are HPV 16, 58, 18, 52, 33, 31, 53, and 56, and the second highest infection rate is HPV 58. In the LSIL and control group, the most prevalent is HPV 52 (Figure 2).

This meta-analysis shows that HPV 16 is the most common infection type among Chinese women with different cervical lesions. The HPV16 infection rates in the ICC, HSIL, LSIL, and normal group are $60.49 \%, 41.77 \%, 18.75 \%$, and $2.39 \%$ respectively, which are higher than the WHO worldwide data, global and Asian meta-analyses [3, 36, 40]. In the study, the five most frequent HR-HPV types with cervical cancer are HPV 16, 58, 18, 52, and 33. Based on WHO worldwide data, the five most frequent HR-HPV types in patients with cervical cancer are HPV 16, 18, 33, 45, and 31. By contrast, WHO data indicate that the five most frequent HPV types in Chinese women cervical cancer are HPV 16, 18, 58, 33, and 52 in 2007 [2]. Clifford et al. determined that the most common genotypes of HPV infection in cervical cancer are HPV 16, 18, 45, 31, and 33 in 2003 [37] (Figure 4). The five most common types of HPV infection in Chinese patients with cervical cancer are HPV $16,18,58,33$, and 52 in the study by Bao et al. in 2008 [41]. The infection rate of HPV 16 and 18 accounted 
for $70.17 \%$ of the total, which is similar to the findings of Bao. Compared with a similar study by Bao et al, changes were also observed in the five most common types of HPV infection in patients with cervical cancer. The results of the study show that HPV 58 and 52 infection rates are higher than Bao's, and HPV18 infection rate has declined. These results are similar to many other studies indicating that HPV 18 is uncommon and that HPV 58 and HPV 52 are more prevalent than HPV 18 in general population and cancer patients in China [42-45].

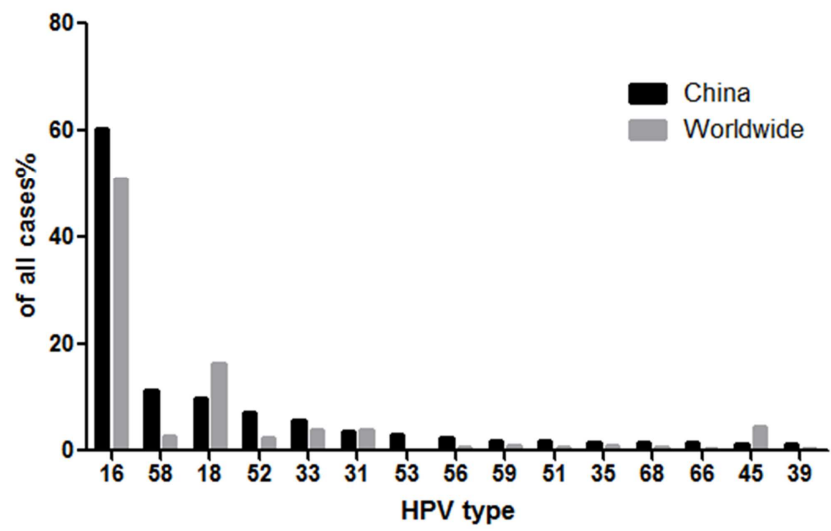

Figure 4. Differences in the HPV infection rates of China and the entire world. This figure shows that the top 10 infection rates of HPV genotypes of China and the entire world are different.

In western countries, HPV 45 is one of the five most frequent HR-HPV genotypes in cervical cancer. In China, the infection rate of HPV 45 is very low in patients with cervical cancer. In addition, the infection rates of the HPV 58 and 52 genotypes in China are higher than those in western countries and the world average. Thus, the national difference should be considered in the prevention and control of HPV and vaccine research in China.

These results indicate that the infection spectrum of HR-HPV types in cervical cancer has changed over the past nine years, which may be due to the difference in the experimental method utilized in the included studies. These differences need further research.

These results show that the HPV infection spectra in cervical cancer are relatively different in different countries and parts of China. Given the geography and population distribution, China can be divided into seven regions (i.e., East China, North China, Central China, South China, Southwest China, Northwest China, and Northeast China). The HPV infection spectra in cervical cancer (Figure 3) show that the most common HPV types in Northeast China are HPV 16, 18, 58, 52 and 31; those in North China are HPV 16, 58, 33, 18and 52; those in East China are HPV 16, 18, 52, 58 and 31; those in South China are HPV 16, 18, 58, 52and 33; those Central China are HPV 16, 58, 68, 52 and 18; those Northwest China are HPV 16, 18, 58, 52 and 33and those in Southwest China are HPV 16, 52, 58, 59 and 18.

The most common genotype is HPV 16, whereas the HPV infection genotypes have clear regional differences. The infection rate of HPV 16 in South China is the highest $(75.56 \%)$, whereas that in Northeast China and East China are lower $(55.07 \%$ and $51.42 \%$, respectively). The second most common genotype is HPV 18 in Northeast China, East China, South China, and Northwest China; HPV 58 in North China and Central China; and HPV 52 in Southwest China. The infection rate of the HPV 18 genotype is highest in East China $(11.09 \%)$. However, its infection rate is not statistically significant compared with that in other regions. The third most common genotype is HPV 58 in Northeast China, South China, Northwest China, and Southwest China; HPV 33 in North China; HPV 52 in East China; and HPV 68 in Central China. The infection rate of HPV 68 in Central China is $7.69 \%$, which is higher than those in Northeast China (1.48\%), North China (1.35\%), East China (0.39\%), and South China (0.89\%). Therefore, the HPV 68 genotype should be given particular attention in developing approaches for prevention and control of HPV infection in Central China (Table 4).

These results indicate that the HPV infection spectra in cervical cancer have significant differences in different nations and regions of China. In particular, HPV 68 ranks third in the infection rate in cervical cancer in Central China but shows an extremely low infection rate in other areas. This phenomenon suggests that HPV68 may be the primary infection genotype of Central China, and the regional differences should be considered when performing HPV prevention, control, and vaccine research in China. The current study has certain differences with that of Chen et al in 2009[39], which shows that the HPV 59 infection rate ranks third in North China and Northwest China. By contrast, this study shows that the HPV 59 infection rate is lower. This scenario suggests that the HPV infection spectra in cervical cancer vary in different regions of China, which may be due to the different experimental methods applied in the included studies. This difference requires further research and observation.

The HPV58 infection rate in seven regions are much higher than other types, which need to pay attention to. This result is similar to the result reported by Chan in 2014 [46]. Therefore, HPV58 genotype may be a kind of high-risk genotype with high infection rate besides HPV 18 and HPV16, suggesting that reducing the infection rate of HPV58 plays a significant role in reducing the occurrence of cervical cancer development. The aforementioned results suggest that the corresponding prevention treatment measures should be taken in the progress of HPV prevention or research and application of vaccines in different parts of China according to the primary infection types in these areas. HPV bivalent, tetravalent, or multivalent vaccines should be researched according to the regional differences and economic states.

As the only vaccine that can prevent malignant tumors thus far, the HPV vaccine is a milestone in the prevention and treatment of cervical cancer [47]. Currently, three HPV vaccines are available worldwide. The bivalent HPV vaccine (Cervarix; GlaxoSmithKline, Boronia, VIC, Australia) can protect against HPV 16 and 18, whereas the quadrivalent HPV (4vHPV) vaccine (Gardasil; CSL, Parkville, VIC, and Merck, Macquarie Park, NSW, Australia) can protect against HPV 6, 11, 16, and 18. A nine-valent HPV (9vHPV) vaccine, which includes HPV types $6,11,16,18,31,33,45,52$, and 58, protecting against the five additional high-risk cancer-causing 
HPV types, was approved by the US Food and Drug Administration in 2014 (Gardasil 9) [48]. Studies in Australia [49-51] showed that the prevalence of HPV 6, 11, 16, and 18 declined significantly for the population that had received the $4 \mathrm{vHPV}$ vaccine. Given the vast territory and large population of China, the HPV vaccine application is highly significant and has broad prospects. However, many developing countries, including China, have failed to adopt the vaccine largely because of its remarkably high price [47]. The 9vHPV vaccine is more expensive than the bivalent HPV vaccine and 4vHPV. Moreover, HPV 45 infection in Chinese patients, which is included in $9 \mathrm{vHPV}$, has much lower prevalence than that in western countries and worldwide. Accordingly, the 9vHPV vaccine is unsuitable for Chinese women.

The development of a HPV vaccine suitable for Chinese population is significant. Particularly, the development of vaccines with regional advantages according to the primary HPV infection types is necessary to improve the preventive and treatment measures in different areas of China.

\section{Conclusion}

HPV 16 is the most common infection type in different cervical lesions, however, the infection rate of HPV 16 genotypes has clear regional differences. HPV 58 and HPV 52 are more prevalent than HPV 18 in general population and cancer patients in China, which need to pay more attention to. Compared to Western countries, the infection rate of HPV 45 is very low in patients with cervical cancer in China. Results of the study show that the HPV infection spectra in cervical cancer have significant differences in different nations and regions of China, This conclusion may be equally adapted to other countries and regions in the world. Therefore, the development of a HPV vaccine suitable for Chinese population is significant which could reduce the burden on the country and the government. Developing vaccines with regional advantages and according to the primary HPV infection types is necessary to improve the preventive and treatment measures in different areas of China, it may be suitable for other countries and regions.

\section{Acknowledgements}

We thank all colleagues who provided us with the information we needed for this study.

\section{Competing Interests}

All authors declare that: there are no significant competing financial, professional or personal interests that might have influenced the performance or presentation of the work described in this manuscript.

\section{Conflict of Interest}

All authors report no conflicts of interest in this publication.

\section{Authors' Contributions}

Yue Wang and Kunpeng Zhang collected all data. Yali Hu participated in the data interpretation. Authors made substantial contributions to conception and design, and/or acquisition of data, and/or analysis and interpretation of data.

\section{References}

[1] Koh WJ, Greer BE, Abu-Rustum NR, Apte SM, Campos SM, Cho KR, et al. Cervical Cancer, Version 2. 2015. J Natl Compr Canc Netw 20152017; 13: 395-404; quiz 404-404.

[2] de Sanjose S, Diaz M, Castellsague X, Clifford G, Bruni L, Munoz N, Bosch FX. Worldwide prevalence and genotype distribution of cervical human papillomavirus DNA in women with normal cytology: a meta-analysis. Lancet Infect Dis 2007;7:453-9.

[3] HPV and cervical cancer in the 2007 report. Vaccine 2007; 25 Suppl 3:C1-230.

[4] Munoz N, Bosch FX, de Sanjose S, Herrero R, Castellsague X, Shah KV, et al. Epidemiologic classification of human papillomavirus types associated with cervical cancer. N. Engl. J. Med. 2003; 348: 518-27.

[5] Li H, Zhang J, Chen Z, Zhou B, Tan Y. Prevalence of human papillomavirus genotypes among women in Hunan province, China. Eur J Obstet Gynecol Reprod BiolEuropean journal of obstetrics, gynecology, and reproductive biology 2013; 170: 202-5.

[6] Li J, Zhang D, Zhang Y, Wang X, Lin Y, Hu L. Prevalence and genotype distribution of human papillomavirus in women with cervical cancer or high-grade precancerous lesions in Chengdu, western China. Int J Gynaecol ObstetInternational journal of gynaecology and obstetrics: the official organ of the International Federation of Gynaecology and Obstetrics 2011; 112: 131-4.

[7] Cai HB, Ding XH, Chen CC. Prevalence of single and multiple human papillomavirus types in cervical cancer and precursor lesions in Hubei, China. OncologyOncology 2009; 76: 157-61.

[8] Wu EQ, Yu XH, Zha X, Zhang GN, Wang JH, Fan Y, et al. Distribution of human papillomavirus genotypes in archival cervical lesions in eastern inner Mongolian autonomous region, China. Int $\mathrm{J}$ Gynecol CancerInternational journal of gynecological cancer: official journal of the International Gynecological Cancer Society 2009; 19: 919-23.

[9] Hong D, Ye F, Chen H, Lu W, Cheng Q, Hu Y, Xie X. Distribution of human papillomavirus genotypes in the patients with cervical carcinoma and its precursors in Zhejiang Province, China. Int J Gynecol CancerInternational journal of gynecological cancer: official journal of the International Gynecological Cancer Society 2008; 18: 104-9.

[10] Zhao Y, Lin H, Shen D, Xuan Y, Lin Z. Distribution of HPV genotypes in uterine cervical lesions in Yanbian, northern China. Pathol IntPathology international 2008; 58: 643-7.

[11] Liu XX, Fan XL, Yu YP, Ji L, Yan J, Sun AH. Human papillomavirus prevalence and type-distribution among women in Zhejiang Province, Southeast China: a cross-sectional study. BMC Infect DisBMC infectious diseases 2014; 14: 708. 
[12] Liang LY, Du H, Wang C, Zhang W, Chen Y, Qu XF, et al. [Crosssectional survey of human papilloma virus subtype distribution and cervical intraepithelial neoplasia in Shenzhen]. Beijing Da Xue Xue BaoBeijing da xue xue bao. Yi xue ban = Journal of Peking University. Health sciences 2013; 45: 114-8.

[13] Liu W, Wu EQ, Yu XH, Feng LH, Jiang CL, Zha X, Kong W. Detection of human papillomavirus genotypes associated with mucopurulent cervicitis and cervical cancer in Changchun, China. Int $\mathrm{J}$ Gynaecol ObstetInternational journal of gynaecology and obstetrics: the official organ of the International Federation of Gynaecology and Obstetrics 2013; 120: $124-6$

[14] Yang L, Li N, Guo LW, Li Q, Cui H, Dai M. [Prevalence of human papilloma virus and analysis of its risk factors in Daqing city, Heilongjiang province in 2010]. Zhonghua Yu Fang Yi Xue Za Zhi 2013; 47: 118-23.

[15] Li J, Mei J, Wang X, Hu L, Lin Y, Yang P. Human papillomavirus type-specific prevalence in women with cervical intraepithelial neoplasm in Western China. J Clin MicrobiolJournal of clinical microbiology 2012; 50: 1079-81.

[16] Yuan X, Yang Y, Gu D, Liu H, Yang H, Wang M. Prevalence of human papillomavirus infection among women with and without normal cervical histology in Shandong Province, China. Arch Gynecol ObstetArchives of gynecology and obstetrics 2011; 283: 1385-9.

[17] Wu D, Cai L, Huang M, Zheng Y, Yu J. Prevalence of genital human papillomavirus infection and genotypes among women from Fujian province, PR China. Eur J Obstet Gynecol Reprod BiolEuropean journal of obstetrics, gynecology, and reproductive biology 2010; 151: 86-90.

[18] Liu X, Zhang S, Ruan Q, Ji Y, Ma L, Zhang Y. Prevalence and type distribution of human papillomavirus in women with cervical lesions in Liaoning Province, China. Int J Gynecol CancerInternational journal of gynecological cancer: official journal of the International Gynecological Cancer Society 2010 20: $147-53$

[19] Sun ZR, Ji YH, Zhou WQ, Zhang SL, Jiang WG, Ruan Q. Characteristics of HPV prevalence among women in Liaoning province, China. Int J Gynaecol ObstetInternational journal of gynaecology and obstetrics: the official organ of the International Federation of Gynaecology and Obstetrics 2010; 109: $105-9$.

[20] Li C, Wu M, Wang J, Zhang S, Zhu L, Pan J, Zhang W. A population-based study on the risks of cervical lesion and human papillomavirus infection among women in Beijing, People's Republic of China. Cancer Epidemiol Biomarkers PrevCancer epidemiology, biomarkers \& prevention: a publication of the American Association for Cancer Research, cosponsored by the American Society of Preventive Oncology 2010;19:2655-64.

[21] Zhang L, Bi Q, Deng H, Xu J, Chen J, Zhang M, Mu X. Human papillomavirus infections among women with cervical lesions and cervical cancer in Eastern China: genotype-specific prevalence and attribution. BMC Infect. Dis. 2017; 17: 107.

[22] Wang Y, Wang S, Shen J, Peng Y, Chen L, Mai R, et al. Genotype Distribution of Human Papillomavirus among Women with Cervical Cytological Abnormalities or Invasive Squamous Cell Carcinoma in a High-Incidence Area of Esophageal Carcinoma in China. Biomed Res Int 2016; 2016: 1256384.

[23] Li J, Wang YY, Nan X, Tian XF, Yan T, Wang P, et al. Prevalence of human papillomavirus genotypes among women with cervical lesions in the Shaanxi Province of China. Genet. Mol. Res. 2016; 15.

[24] Xiao M, Xu Q, Li H, Gao H, Bie Y, Zhang Z. Prevalence of Human Papillomavirus Genotypes Among Women With High-Grade Cervical Lesions in Beijing, China. Medicine (Baltimore) 2016; 95: e2555.

[25] Wang L, Wang P, Ren Y, Du J, Jiang J, Jia X, et al. Prevalence of High-Risk Human Papillomavirus (HR-HPV) Genotypes and Multiple Infections in Cervical Abnormalities from Northern Xinjiang, China. PLoS ONE 2016; 11: e0160698.

[26] Baloch Z, Li Y, Yuan T, Feng Y, Liu Y, Tai W, et al. Epidemiologic characterization of human papillomavirus (HPV) infection in various regions of Yunnan Province of China. BMC Infect. Dis. 2016; 16: 228.

[27] Feng YK, Peng Y, Zhu L, Niu XY. [Relationship of Human Papillomavirus Subtypes and Multiple Infection with Different Cervical Precancerous Diseases in Sichuan Province]. Sichuan Da Xue Xue Bao Yi Xue BanSichuan da xue xue bao. Yi xue ban $=$ Journal of Sichuan University. Medical science edition 2015; 46: 422-5, 462.

[28] Ma L, Cong X, Bian M, Shi M, Wang X, Liu J, Liu H. [High-risk HPV genotyping PCR testing as a means of cervical cancer and precancerous lesions early screening]. Zhonghua $\mathrm{Fu}$ Chan Ke Za ZhiZhonghua fu chan ke za zhi 2015; 50: 246-52.

[29] Ma Q, Hou M, Yang XF. Screening of the genital human papillomavirus infection among 8581 women in the First Affiliated Hospital of Xi'an Jiaotong University. Zhongguo Yi Xue Ke Xue Yuan Xue BaoZhongguo yi xue ke xue yuan xue bao. Acta Academiae Medicinae Sinicae 2014; 36: 277-82.

[30] Ding X, Liu Z, Su J, Yan D, Sun W, Zeng Z. Human papillomavirus type-specific prevalence in women referred for colposcopic examination in Beijing. J Med VirolJournal of medical virology 2014; 86: 1937-43.

[31] Li K, Yin R, Li Q, Wang D. Analysis of HPV distribution in patients with cervical precancerous lesions in Western China. Medicine (Baltimore) 2017;96:e7304.

[32] Li Y, Huang K, Ji PL, Song L, Liu HT. Cervical Infection of Oncogenic Human Papillomavirus (HPV) Types in Beijing, China. Biomedical and environmental sciences: BES 2016; 29: 734-41.

[33] Mai RQ, Huang B, Shen L, Zhang GH, Hong LL, Cai YM. Genotype distribution of human papillomavirus in women with abnormal cervical cytology in an esophageal carcinoma high incidence area of China. Asian Pac J Cancer PrevAsian Pacific journal of cancer prevention: APJCP 2014; 15: 4945-50.

[34] Distribution of human papippomavirus types in cervical cancer of Xinjiang Uyghur women. Journal of International Oncology 2011; 38: 237-40.

[35] Shui-ping H. The method of multiple comparisons of rate. Acta Academiae Medicinae Xuzhou 2002; 291-4.

[36] JS S, L L, B H, Al E. Human papillomavirus type distributiondistributionin invasive cervical cancerand high-grade cervical lesions:ameta-analysis update. Int. J. Cancer 2007; 121: 621-32.

[37] Clifford GM, Smith JS, Plummer M, Munoz N, Franceschi S. Human papillomavirus types in invasive cervical cancer worldwide: a meta-analysis. Br. J. Cancer 2003; 88: 63-73. 
[38] GM C, JS S, T A, Al E. Comparison of HPV type distributionin high-grade cervical lesionsand cervical cancer:a metaanalysis. Br. J. Cancer 2003; 89: 101-5.

[39] Chen W, Zhang X, Molijn A, Jenkins D, Shi JF, Quint W, et al. Human papillomavirus type-distribution in cervical cancer in China: the importance of HPV 16 and 18. Cancer Causes Control 2009; 20: 1705-13.

[40] Bhatla N, Lal N, Bao YP, Ng T, Qiao YL. A meta-analysis of human papillomavirus type-distribution in women from South Asia: implications for vaccination. Vaccine 2008; 26: 2811-7-.

[41] Bao YP, Li N, Smith JS, Qiao YL. Human papillomavirus type-distribution in the cervix of Chinese women: a meta-analysis. Int J STD AIDS 2008; 19: 106-11-.

[42] Wang L, Wu B, Li J, Chen L. Prevalence of human papillomavirus and its genotype among 1336 invasive cervical cancer patients in Hunan province, central south China. J. Med. Virol. 2015; 87: 516-21.

[43] Jing L, Zhong X, Huang W, Liu Y, Wang M, Miao Z, et al. HPV genotypes and associated cervical cytological abnormalities in women from the Pearl River Delta region of Guangdong province, China: a cross-sectional study. BMC Infect. Dis. 2014; 14: 388.

[44] Wu EQ, Liu B, Cui JF, Chen W, Wang JB, Lu L, et al. Prevalence of type-specific human papillomavirus and pap results in Chinese women: a multi-center, population-based cross-sectional study. Cancer Causes Control 2013; 24: 795-803.
[45] Li J, Kang LN, Qiao YL. Review of the cervical cancer disease burden in mainland China. Asian Pac. J. Cancer Prev. 2011; 12: 1149-53.

[46] Chan PK, Ho WC, Chan MC, Wong MC, Yeung AC, Chor JS, Hui M. Meta-analysis on prevalence and attribution of human papillomavirus types 52 and 58 in cervical neoplasia worldwide. PLoS ONE 2014;9:e107573-107573.

[47] Zhang WJ, Li F, Wang YH, Simayi D, Saimaiti A, Zou XG, et al. The case for semi-mandatory HPV vaccination in China. Nat. Biotechnol. 2013; 31: 590-1.

[48] US Food and Drug Administration. Gardasil 9. http://www.fda.gov/BiologicsBloodVaccines/Vaccines/Approv edProducts/ucm426445.

[49] EPF C, Machalek DA, Tabrizi SN, Danielewski JA, Fehler G, Bradshaw CS, et al. Quadrivalent vaccine-targeted human papillomavirus genotypes in heterosexual men after the Australian female human papillomavirus vaccination programme: a retrospective observational study. Lancet Infect Dis 2017; 17: 68-77.

[50] Chow EP, Danielewski JA, Fehler G, Tabrizi SN, Law MG, Bradshaw CS, et al. Human papillomavirus in young women with Chlamydia trachomatis infection 7 years after the Australian human papillomavirus vaccination programme: a cross-sectional study. Lancet Infect Dis 2015; 15: 1314-23.

[51] Brotherton JM, Ogilvie GS. Current status of human papillomavirus vaccination. Curr Opin Oncol 2015; 27: 399-404. 